\title{
Controle Descentralizado de Sistemas Interconectados por Não-Linearidades Setoriais Sujeitos a Saturação nos Atuadores
}

\author{
Rodrigo F. Araújo* Leonardo A. B. Torres ${ }^{* *}$ \\ Reinaldo M. Palhares ** \\ * Programa de Pós-Graduação em Engenharia Elétrica - Universidade \\ Federal de Minas Gerais - Av. Antônio Carlos 6627, 31270-901, Belo \\ Horizonte, MG, Brasil (e-mail: rodfaraujo@gmail.com) \\ ** Departamento de Engenharia Eletrônica, Universidade Federal de \\ Minas Gerais - Av. Antônio Carlos 6627, 31270-901, Belo Horizonte, \\ MG,Brasil (e-mail: leotorres@ufmg.br, rpalhares@ufmg.br)
}

\begin{abstract}
The problem of designing decentralized control laws for continuous-time interconnected systems through sector-boundedness nonlinearities subject to state and control input constraints is addressed in this paper. Sufficient conditions taking into account state constraints are derived and expressed in terms of Linear Matrix Inequalities (LMIs). It is shown that the closed-loop system can be made asymptotically stable while at the same time, the estimate of the domain of attraction for the closed-loop system is maximized. Finally, the synchronization problem of an electric distribution network composed by generators is considered in order to illustrate the effectiveness of the design approach proposed.

Resumo: Neste artigo aborda-se o problema de síntese de leis de controle descentralizadas para sistemas interconectados a tempo contínuo com não-lineares setoriais e sujeitos a restrições nos estados e na entrada de controle. Obtém-se condições suficientes que levam em conta restrições no estado e no controle descritas na forma de Desigualdades Matriciais Lineares (LMIs). Mostra-se que o sistema em malha fechada pode ser assintoticamente estável, enquanto, ao mesmo tempo, a estimativa da região de atração para o sistema de malha fechada é maximizada. Para ilustrar a eficácia da abordagem proposta, considera-se o problema de sincronismo de uma rede de distribuição elétrica composta por geradores de energia.
\end{abstract}

Keywords: Decentralized Control; Interconnected Systems; Sector Nonlinearity; Saturation; Lyapunov Theory.

Palavras-chaves: Controle Descentralizado; Sistemas Interconectados; Não-linearidade Setorial; Saturação; Teoria de Lyapunov.

\section{INTRODUÇÃO}

Tendo em vista que vários sistemas de grande escala são interconectados como, por exemplo, sistemas de distribuição de energia, água, gás, processos industriais, enxame de robôs móveis ou UAVs, sistemas de tráfego urbano, dentre outros, aprimorar o seu controle é um tópico de grande interesse tanto por aspectos econômicos quanto em relação a aspectos de segurança para o seu funcionamento. Devido a uma possível alta dimensionalidade, a interconexões incertas e não-lineares ou ainda à possível presença de atraso de tempo (Šiljak, 2007; Mahmoud, 2010), obter técnicas para análise de estabilidade de sistemas interconectados pode ser bastante desafiador, o que conduz muitas vezes a abordagens que são conservadoras, de pouca abrangência. Particularmente, no contexto de interconexões não-lineares o desafio é ainda maior.

Uma das técnicas mais comuns para lidar com nãolinearidades é o uso de modelos fuzzy Takagi-Sugeno (T-S), já que tais modelos podem aproximar funções não lineares contínuas com precisão arbitrária (Takagi and Sugeno, 1985; Nguyen et al., 2019). Contudo, se cada interconexão não-linear for representada por um conjunto de regras fuzzy, pode-se ter uma explosão do número de regras e, consequentemente, da complexidade computacional. Outras estratégias empregadas no contexto de interconexões não-lineares incluem fazer uso de condições de Lipschitz (Li et al., 2018), ou ainda decompor a interconexão nãolinear em uma combinação de um termo linear e um outro termo linear incerto (Chang et al., 2014).

O controle centralizado de tais sistemas pode não ser viável, tendo em vista a demanda de comunicação entre a estrutura central e todos os subsistemas envolvidos, que pode ser expressiva, bem como a complexidade computacional desta estrutura centralizadora, principalmente quando há um número significativo de subsistemas. Como alternativa, métodos de controle descentralizado tem sido aplicados com sucesso, sempre que é possível dividir o sistema de grande escala em subsistemas independentes ou quase independentes, cada um controlado por ações de 
controle locais que dependem apenas dos estados do subsistema em questão, e eventualmente de informações das interconexões com seus vizinhos (veja, por exemplo, (Šiljak and Zečević, 2005; Bakule, 2008, 2014) e suas referências). O que se espera é que, ao se garantir estabilidade local dos subsistemas, se alcance estabilidade global do sistema interconectado.

Outra característica frequentemente presente em sistemas reais é estarem sujeitos a restrições nos estados e na entrada de controle devido, por exemplo, a restrições físicas, restrições econômicas, ou por questões de segurança (Nguyen et al., 2016). Destaca-se ainda o caso em que o modelo do sistema é válido apenas em uma determinada região do espaço de estados representada por restrições a serem obedecidas. Ao se levar em consideração tais restrições na síntese de controladores, é possível garantir que as trajetórias do sistema irão evoluir dentro da região de validade do modelo utilizado, mantendo a consistência da análise realizada. Por outro lado, restrições nas entradas de controle ou saturação em atuadores surgem da limitação dos atuadores do sistema e podem degradar consideravelmente o desempenho em malha fechada, podendo levar o sistema a condições críticas de funcionamento ou mesmo à instabilidade, se não forem consideradas no projeto de controle (Tarbouriech et al., 2011). Este tema tem sido explorado tanto em sistemas a tempo contínuo quanto a tempo discreto (Castelan et al., 2008; Gonzaga et al., 2012).

Os trabalhos mencionados anteriormente utilizam o método direto de Lyapunov para produzir condições suficientes para verificação de estabilidade e síntese de controladores, as quais são normalmente expressas na forma de desigualdades matriciais lineares (LMIs) (Boyd et al., 1994). Nesse contexto, determinar exatamente a região de atração pode ser muito difícil, e frequentemente apenas a estabilidade regional pode ser garantida, com base em uma estimativa da região de atração.

Neste artigo aborda-se o problema de síntese de controladores descentralizados para uma classe de sistemas a tempo contínuo, interconectados, e sujeitos a restrições nos estados e na entrada de controle. O sistema é composto de subsistemas localmente lineares com saturação nos atuadores e interconectados via não-linearidades setoriais, as quais são representadas por um grafo bidirecional como em (Khalili et al., 2018). A partir da escolha de uma função de Lyapunov quadrática, condições suficientes, expressas na forma de LMIs, são obtidas. Além disso, um dos objetivos é maximizar o volume da estimativa da região de atração do sistema em malha fechada.

O restante deste artigo é organizado da seguinte forma. Os conceitos básicos da teoria de grafos, não-linearidades setoriais e saturação na entrada de controle são introduzidos na Seção 2. A formulação do problema é apresentada na Seção 3. Na Seção 4 são obtidas condições suficientes para estabilização, além da formulação do problema de maximização do volume da estimativa da região de atração. Para ilustrar a efetividade do método proposto, uma rede de distribuição de energia formada apenas por geradores é utilizada na Seção 5. Finalmente, na Seção 6 apresenta-se as conclusões.
Notação: Dada uma matriz $X=\left[x_{i j}\right]$ com dimensões arbitrárias, a l-ésima linha é denotada por $X_{(l)} \cdot X^{\top}$ é sua transposta. $X \succ 0(X \succeq 0)$ significa que $X=X^{\top}$ e $X$ é uma matriz (semi-)definida positiva. O símbolo ' $\star$ ' denota blocos simétricos em uma matriz simétrica. $\mathcal{I}_{n} \triangleq\{1, \ldots, n\} \subset \mathbb{N}$ é um conjunto de índices. $\mathbf{1}_{N} \mathrm{e}$ $\mathbf{0}_{N}$ são vetores colunas com $N$ elementos iguais a 1 e 0 , respectivamente. Além disso, sejam matrizes $X_{i}$ com dimensões arbitrárias, $\mathbf{X}_{N}$ é uma matriz bloco diagonal de matrizes $X_{i}, \forall i \in \mathcal{I}_{N}$, ou seja, $\mathbf{X}_{N}=\operatorname{diag}\left(Y_{1}, \ldots, Y_{N}\right)$. Adicionalmente, o operador ' $\circ$ ' denota o produto de Hadamard entre matrices. Por simplicidade, a dependência das variáveis com o tempo $t$ é omitida ao longo deste artigo, quando seu sentido for direto.

\section{PRELIMINARES}

Nesta seção são apresentados conceitos básicos da teoria de grafos, não-linearidades setoriais e saturação na entrada de controle.

\subsection{Teoria de Grafos}

A teoria de grafos fornece uma estrutura adequada para representar as relações entre sistemas interconectados em rede. Um grafo $\mathcal{G}(\mathcal{V}, \mathcal{E})$ é composto por um conjunto de vértices $\mathcal{V} \triangleq\{1, \ldots, N\}$ e um conjunto de interconexões $\mathcal{E} \triangleq\{(i, j): i, j \in \mathcal{V}\}$. Dado um grafo bidirecional $\mathcal{G}$, sua matriz de adjacência $\mathcal{A}=\left[\alpha_{i j}\right] \in \mathbb{R}^{N \times N}$ é tal que $\alpha_{i j}=1$, se $(i, j) \in \mathcal{E}$, indicando uma interconexão entre o $i$-ésimo e o $j$-ésimo vértices e, caso contrário, $\alpha_{i j}=0$. Uma vez que o grafo é bidirecional, a matriz de adjacência é simétrica. Além disso, a vizinhança de subsistemas interconectados ao $i$-ésimo subsistema é definido por um conjunto de índices $\mathcal{N}_{i}=\left\{j \in \mathcal{V}: \alpha_{i j} \neq 0\right\}$.

A matriz de grau de um grafo, $\mathcal{D}=\left[d_{i j}\right] \in \mathbb{R}^{N \times N}$, é uma matriz diagonal que contém informações dos números de conexões associadas a cada vértice, tal que $d_{i i}=\sum_{j=1}^{N} \alpha_{i j}$ $\forall i \in \mathcal{V}$; e $d_{i j}=0$ se $i \neq j$. A partir das matrizes de adjacência e grau, pode-se obter a matriz Laplaciana $\mathcal{L}=\left[l_{i j}\right] \in \mathbb{R}^{N \times N}$ do grafo: $\mathcal{L} \triangleq \mathcal{D}-\mathcal{A}$. Uma vez que as matrizes de adjacência e grau de um grafo bidirecional são simétricas, a matriz Laplaciana também é simétrica e satisfaz $\mathcal{L} \mathbf{1}_{N}=\mathbf{0}_{N}$; i.e. $\sum_{j=1}^{N} l_{i j}=0, \forall i \in \mathcal{V}$.

\subsection{Não-Linearidade Setorial e Saturação}

Ao longo deste artigo faremos uso de dois tipos de nãolinearidades: não-linearidade setorial; e saturação representada por uma não-linearidade do tipo zona-morta. Dessa maneira, os Lemas a seguir serão instrumentais.

Lema 1. (Khalil, 2002) Um vetor de funções não-lineares, $\phi(x)$, é dito ser uma não-linearidade setorial se satisfaz a seguinte condição:

$$
\phi(x)^{\top} \Lambda^{-1}(\phi(x)-E x) \leq 0,
$$

$\forall x \in \mathbb{R}^{n_{x}}$, com $E=\left[\begin{array}{llll}E_{(1)}^{\top} & E_{(2)}^{\top} & \cdots & E_{\left(n_{\phi}\right)}^{\top}\end{array}\right]^{\top} \in \mathbb{R}^{n_{\phi} \times n_{x}}$ conhecida, e $\Lambda \in \mathbb{R}^{n_{\phi} \times n_{\phi}}$ uma matriz diagonal definida positiva. 
Considere um sistema a tempo contínuo sujeito a saturação na entrada de controle, dado por:

$$
\dot{x}=A x+B \operatorname{sat}(u) \text {. }
$$

A saturação pode ser escrita como uma não-linearidade do tipo zona-morta da forma: $\psi(u)=u-\operatorname{sat}(u)$. Assim, para $u=K x$ (controle por realimentação linear de estados), pode-se considerar o Lema a seguir.

Lema 2. (Tarbouriech et al., 2011) Dada uma matriz $W \in \mathbb{R}^{n_{u} \times n_{x}}$, define-se o seguinte conjunto:

$$
\mathcal{D}_{u}=\left\{x \in \mathbb{R}^{n_{x}}:\left|(K-W)_{(l)} x\right| \leq \bar{u}_{(l)}, \forall l \in \mathcal{I}_{n_{u}}\right\} \text {. }
$$

Se $x \in \mathcal{D}_{u}$, então

$$
\psi(u)^{\top} U^{-1}(\psi(u)-W x) \leq 0,
$$

é satisfeita para qualquer matriz diagonal definida positiva $U \in \mathbb{R}^{n_{u} \times n_{u}}$.

\section{FORMULAÇÃO DO PROBLEMA}

Considere um sistema interconectado, a tempo contínuo, composto por $N$ subsistemas sujeitos a saturação na entrada de controle e interconectados por não-linearidades setoriais. $\mathrm{O} i$-ésimo subsistema é descrito como:

$$
\dot{x}_{i}(t)=A_{i} x_{i}(t)+B_{i} \operatorname{sat}\left(u_{i}(t)\right)+G_{i} \sum_{j \in \mathcal{N}_{i}} \varphi_{i j}(x(t)),
$$

em que $i \in \mathcal{I}_{N}, x_{i}(t) \in \mathbb{R}^{n_{x_{i}}}$ é o vetor de estados do $i$-ésimo subsistema; $u_{i}(t) \in \mathbb{R}^{n_{u_{i}}}$ é o $i$-ésimo vetor de entrada sujeito a saturação, tal que $\operatorname{sat}\left(u_{i(l)}(t)\right)=$ $\max \left(\min \left(u_{i(l)}(t), \bar{u}_{i(l)}\right),-\bar{u}_{i(l)}\right), \forall l \in \mathcal{I}_{n_{u_{i}}}, \operatorname{com} \bar{u}_{i(l)}>0$ sendo o limite superior da $l$-ésima entrada de controle do $i$-ésimo subsistema; $\varphi_{i j}(\cdot): \mathbb{R} \rightarrow \mathbb{R}$ são funções descrevendo as interconexões não-lineares entre o $i$-ésimo e $j$-ésimo subsistemas. $A_{i}, B_{i}$, e $G_{i}$ são matrizes constantes e conhecidas de dimensões apropriadas, com $G_{i}$ ponderando as contribuições dos acoplamentos não-lineares.

A partir da descrição em (5), o vetor de estados do sistema global é dado por $x(t)=\left[\begin{array}{lll}x_{1}^{\top}(t) & \cdots & x_{N}^{\top}(t)\end{array}\right]^{\top} \in \mathbb{R}^{n_{x}}$, com $n_{x}=\sum_{i=1}^{N} n_{x_{i}}$, tal que a dinâmica do sistema global pode ser expressa como

$$
\dot{x}(t)=\mathbf{A}_{N} x(t)+\mathbf{B}_{N} \operatorname{sat}(u(t))+\mathbf{G}_{N}\left[(\mathcal{A} \circ \Phi(x(t))) \mathbf{1}_{N}\right],
$$

em que $\operatorname{sat}(u)=\left[\operatorname{sat}\left(u_{1}(t)\right)^{\top} \cdots \operatorname{sat}\left(u_{N}(t)\right)^{\top}\right]^{\top} ; \mathbf{A}_{N}$, $\mathbf{B}_{N}$ e $\mathbf{G}_{N}$ são matrizes de blocos diagonal; $\Phi(x(t))=$ $\left[\varphi_{i j}(x(t))\right] \in \mathbb{R}^{N \times N}$ é uma matriz de funções não-lineares; $\mathcal{A}$ é a matriz de adjacência associada com o grafo que representa as interconexões entre os subsistemas.

Para se evitar problemas numéricos nas condições de síntese apresentadas posteriormente, faze-se necessário excluir as não-linearidades que não existem, ou seja, quando $\alpha_{i j}=0$. Assim, defini-se um vetor $\phi_{i}(x(t))=$ $\left[\varphi_{i j_{1}}(t) \varphi_{i j_{2}}(t) \cdots \varphi_{i j_{i i}}(t)\right]^{\top} \in \mathbb{R}^{d_{i i}}$ como um vetor coluna formado pelo empilhamento das funções não-lineares $\varphi_{i j_{\kappa}}(x(t))$, com $j_{\kappa} \in \mathcal{N}_{i}$, o qual corresponde as funções não-lineares que descrevem as interconexões do $i$-ésimo subsistema com seus vizinhos.

Observação 1 . Note que se cada função $\varphi_{i j}(x)$ é uma nãolinearidade setorial a partir da definição de $\phi_{i}(x)$, esta é uma não-linearidade setorial que satisfaz o Lema 1 , ou seja, existe uma determinada matriz $E_{i}=\left[\begin{array}{llll}E_{i 1} & E_{i 2} & \cdots & E_{i N}\end{array}\right] \in$ $\mathbb{R}^{d_{i i} \times n_{x}}$, em que $E_{i j} \in \mathbb{R}^{d_{i i} \times n_{x_{j}}}, \forall j \in \mathcal{I}_{N}$, tal que (1) é satisfeita para qualquer matriz diagonal definida positiva $\Lambda_{i} \in \mathbb{R}^{d_{i i} \times d_{i i}}$.

Adicionalmente, será considerado na síntese de controladores descentralizados que o modelo em (6) é válido apenas em uma determinada região poliedral do espaço de estados, dada por

$$
\mathcal{D}_{x}=\left\{x \in \mathbb{R}^{n_{x}}: \beta_{\nu}^{\top} x \leq 1, \nu \in \mathcal{I}_{n_{e}}\right\},
$$

em que $\beta_{\nu} \in \mathbb{R}^{n_{x}}$ são vetores conhecidos, e $n_{e}$ é o número de restrições a serem satisfeitas.

Para a síntese de controle, serão consideradas leis de controle descentralizado para cada $i$-ésimo subsistema, tais que:

onde $K_{i} \in \mathbb{R}^{n_{u_{i}} \times n_{x_{i}}}$

$$
u_{i}(t)=K_{i} x_{i}(t)
$$

Substituindo (8) em (5) e usando $\phi_{i}(x)$ definida anteriormente, temos que o $i$-ésimo subsistema em malha fechada é dado por:

$$
\dot{x}_{i}(t)=\left(A_{i}+B_{i} K_{i}\right) x_{i}(t)-B_{i} \psi_{i}\left(u_{i}(t)\right)+G_{i} \mathbf{1}_{d_{i i}}^{\top} \phi_{i}(x(t)),
$$

em que $\psi_{i}\left(u_{i}(t)\right)=u_{i}(t)-\operatorname{sat}\left(u_{i}(t)\right)$ é uma não-linearidade do tipo zona-morta que satisfaz o Lema 2.

Observação 2. Note que, $\psi(x)=\left[\psi_{1}(x)^{\top} \ldots \psi_{N}(x)^{\top}\right]^{\top}$ também satisfaz o Lema 2 , para $\mathbf{K}_{N}$ e $W \in \mathbb{R}^{n_{u} \times n_{x}}$, e $U \in \mathbb{R}^{n_{u} \times n_{u}} \operatorname{com} n_{u}=\sum_{i=1}^{N} n_{u_{i}}$.

Considere, para a análise de estabilidade dos $N$ subsistemas interconectados em malha fechada, representados em (9), a função de Lyapunov candidata a seguir, composta pelo somatório de funções quadráticas:

$$
V(x(t))=\sum_{i=1}^{N} x_{i}(t)^{\top} P_{i} x_{i}(t)=x(t)^{\top} \mathbf{P}_{N} x(t) .
$$

Observação 3. A região elipsodial associada com a função quadrática (10) é definida como $\mathcal{E}_{V}=\left\{x(t) \in \mathbb{R}^{n_{x}}\right.$ : $\left.x(t)^{\top} \mathbf{P}_{N} x(t) \leq 1\right\}$. Além disso, se $\dot{V}(x(t))<0$ ao longo das trajetórias do sistema não-linear composto pelos sistemas interconectados $(9), \forall x(t) \in \mathcal{E}_{V} \backslash\{0\}$, então (10) é chamada de função de Lyapunov e $\mathcal{E}_{V}$ é um conjunto elipsoidal positivamente invariante, consequentemente também é um subconjunto da região de atração do sistema em malha fechada (Khalil, 2002).

Baseado nas discussões anteriores, o objetivo deste artigo é propor um procedimento de síntese de controladores descentralizados para resolver o seguinte problema.

Problema 1. Projetar controladores descentralizados (8) para cada $i$-ésimo subsistema, tal que o volume da estimativa da região de atração, $\mathcal{E}_{V} \subset \mathcal{D}_{x} \cap \mathcal{D}_{u}$, do sistema global em malha fechada, composto por subsistemas interconectados (9), seja maximizado.

\section{CONDIÇÕES DE ESTABILIZAÇÃO DECENTRALIZADA PARA SISTEMAS INTERCONECTADOS}

O Teorema 1 a seguir fornece condições suficientes para garantir que a origem do sistema não-linear em malha fechada formado pelos subsistemas em (9) seja assintoticamente estável. 
Teorema 1. O sistema a tempo contínuo (5), composto por $N$ subsistemas interconectados, pode ser feito assintoticamente estável por meio de leis de controle descentralizado (8), se existirem matrizes definidas positivas $Q_{i} \succ 0$, matrizes diagonais definidas positivas $\Lambda_{i} \succ 0$ e $U \succ 0$, e quaisquer matrizes $W$ e $R_{i}, \forall i \in \mathcal{I}_{N}$, que satisfaçam o seguinte conjunto de LMIs:

$$
\begin{aligned}
& {\left[\begin{array}{cc}
1 & \star \\
\mathbf{Q}_{N} \beta_{\nu} & \mathbf{Q}_{N}
\end{array}\right] \succeq 0, \quad \forall \nu \in \mathcal{I}_{n_{e}},} \\
& {\left[\begin{array}{ccc}
\mathbf{Q}_{N} & \star & \star \\
-\bar{E} \mathbf{Q}_{N} & 2 \boldsymbol{\Lambda}_{N} & \star \\
\left(\mathbf{R}_{N}-W\right)_{(l)} & 0 & \bar{u}_{(l)}^{2}
\end{array}\right] \succ 0, \forall l \in \mathcal{I}_{n_{u}}} \\
& {\left[\begin{array}{ccc}
\boldsymbol{\Theta}_{N} & \star & \star \\
\mathbf{\Pi}_{N}+\bar{E} \mathbf{Q}_{N} & -2 \boldsymbol{\Lambda}_{N} & \star \\
-U \mathbf{B}_{N}^{\top}+W & 0 & -2 U
\end{array}\right] \prec 0,}
\end{aligned}
$$

em que, $\Theta_{i}=Q_{i} A_{i}^{\top}+A_{i} Q_{i}+R_{i}^{\top} B_{i}^{\top}+B_{i} R_{i}, \Pi_{i}=\Lambda_{i} \mathbf{1}_{d_{i i}} G_{i}^{\top}$, $\bar{E}=\left[\begin{array}{llll}E_{1}^{\top} & E_{2}^{\top} & \cdots & E_{N}^{\top}\end{array}\right]^{\top}$.

Os ganhos dos controladores em (8) podem ser recuperados através de $K_{i}=R_{i} Q_{i}^{-1}$.

Prova. Assuma que (11) é satisfeita. Aplicando a transformação de congruência $\operatorname{diag}\left(1, \mathbf{P}_{N}\right), \operatorname{com} P_{i}=Q_{i}^{-1}$, $\forall i \in \mathcal{I}_{N}$, i.e. $\mathbf{P}_{N}=\mathbf{Q}_{N}^{-1}$, tem-se que

$$
\left[\begin{array}{cc}
1 & \star \\
\beta_{\nu} & \mathbf{P}_{N}
\end{array}\right] \succeq 0
$$

Pré- e pós-multiplicando a desigualdade acima por $\left[\begin{array}{ll}1 & -x^{\top}\end{array}\right]$ e sua transposta, respectivamente, resulta em:

$$
1-x^{\top} \beta_{\nu}-\beta_{\nu}^{\top} x+x^{\top} \mathbf{P}_{N} x \geq 0 .
$$

Uma vez que $x^{\top} \mathbf{P}_{N} x \leq 1, \forall x \in \mathcal{E}_{V}$, então $2-x^{\top} \beta_{\nu}-$ $\beta_{\nu}^{\top} x \geq 0$ é satisfeita $\forall x \in \mathcal{E}_{V}$, o que implica que $\beta_{\nu}^{\top} x \leq 1$. Consequentemente, $\mathcal{E}_{V} \subseteq \mathcal{D}_{x}$, com $\mathcal{D}_{x}$ definido em (7). Aplicando a transformação de congruência $\operatorname{diag}\left(\mathbf{P}_{N}, \boldsymbol{\Lambda}_{N}^{-1}, 1\right)$ em (12), seguida do complemento de Schur, obtém-se

$$
\left[\begin{array}{cc}
\mathbf{P}_{N} & \star \\
-\boldsymbol{\Lambda}_{N}^{-1} \bar{E} & 2 \boldsymbol{\Lambda}_{N}^{-1}
\end{array}\right]-\frac{1}{\bar{u}_{(l)}^{2}} \Upsilon_{(l)}^{\top} \Upsilon_{(l)} \succ 0, \quad \forall l \in \mathcal{I}_{n_{u}}
$$

em que, $\Upsilon=\mathbf{K}_{N}-\tilde{W}$, com $\tilde{W}=W \mathbf{P}_{N}$. Então pré- e pós-multiplicando (14) por $\left[x^{\top} \phi(x)^{\top}\right]$ e sua transposta, respectivamente, além de usar (10) como função de Lyapunov, tem-se que

$$
\begin{aligned}
V(x)+2 \sum_{i \in \mathcal{I}_{N}} \phi_{i}(x)^{\top} \Lambda_{i}^{-1}\left(\phi_{i}(x)-E_{i} x\right) & \\
- & \frac{\left\|\left(\mathbf{K}_{N}-\tilde{W}\right)_{(l)} x\right\|^{2}}{\bar{u}_{(l)}^{2}}>0 .
\end{aligned}
$$

Assim, utilizando os Lemas 1 e 2, pode-se concluir a partir de (15) que $\mathcal{E}_{V} \subset \mathcal{D}_{u}$, com $\mathcal{D}_{u}$ definido em (3). Finalmente, se a inequação (13) é satisfeita, tomando $R_{i}=K_{i} P_{i}, \forall i \in \mathcal{I}_{N}$, e aplicando a transformação de congruência diag $\left(\mathbf{P}_{N}, \boldsymbol{\Lambda}_{N}^{-1}, U^{-1}\right)$, tem-se a seguinte desigualdade matricial

$$
\left[\begin{array}{ccc}
\overline{\boldsymbol{\Theta}}_{N} & \star & \star \\
\overline{\mathbf{\Pi}}_{N}+\boldsymbol{\Lambda}_{N}^{-1} \bar{E} & -2 \boldsymbol{\Lambda}_{N}^{-1} & \star \\
-\mathbf{B}_{N}^{\top} \mathbf{P}_{N}+U^{-1} W \mathbf{P}_{N} & 0 & -2 U^{-1}
\end{array}\right] \prec 0
$$

$\operatorname{com} \bar{\Theta}_{i}=A_{i}^{\top} P_{i}+P_{i} A_{i}+K_{i}^{\top} B_{i}^{\top}+B_{i} K_{i}$ e $\bar{\Pi}_{i}=\mathbf{1}_{d_{i i}} G_{i}^{\top} P_{i}$. Pré- e pós-multiplicando (16) por $\left[x^{\top} \phi(x)^{\top} \psi(u)^{\top}\right]$ e sua transposta, respectivamente, e novamente considerando (10), tem-se que

$$
\begin{aligned}
\dot{V}(x)-2 \sum_{i \in \mathcal{I}_{N}} \phi_{i}(x)^{\top} \Lambda_{i}^{-1}\left(\phi_{i}(x)-E_{i} x\right) & \\
& -2 \psi(u)^{\top} U^{-1}(\psi(u)-\tilde{W} x)<0 .
\end{aligned}
$$

Dessa forma, como cada não-linearidade $\phi_{i}(x)$ satisfaz o Lema 1 e $\psi(u)$ é uma não-linearidade do tipo zona-morta, que satisfaz o Lema 2, a desigualdade (17) define um limite superior para a derivada temporal da função de Lyapunov (10), implicando que $\dot{V}(x)<0, \forall x \neq 0$. Desta forma, se conclui a demonstração.

O Teorema 1 fornece condições suficientes para guarantir estabilidade assintótica e uma estimativa da região de atração, $\mathcal{E}_{V}$, dos sistemas interconectados. Contudo, podese também encontrar a maior estimativa considerando $\mathcal{D}_{x}$ e $\mathcal{D}_{u}$, isto é $\mathcal{E}_{V} \subset \mathcal{D}_{x} \cap \mathcal{D}_{u}$. O Corolário 2, dado a seguir, soluciona o Problema 1 ao incorporar a maximização do volume $\mathcal{E}_{V}$, enquanto considera as condições suficientes do Teorema 1 .

Corolário 2. Se o problema de otimização,

$$
\begin{aligned}
& \max _{Q_{i}, R_{i}, \Lambda_{i}, W, U} \operatorname{det}\left(\mathbf{Q}_{N}\right)^{1 / n_{x}} \\
& \text { sujeito a: (11), (12) e (13). }
\end{aligned}
$$

é factível, então o Problema 1 tem uma solução que fornece o maior conjunto elipsoidal positivamente invariante $\mathcal{E}_{V}$ sob as condições consideradas.

Prova. Note que maximizar $\operatorname{det}\left(\mathbf{Q}_{N}\right)^{1 / n_{x}}$ implica em minimizar det $\left(\mathbf{P}_{N}\right)^{1 / n_{x}}$ e consequentemente o volume do conjunto $\mathcal{E}_{V}$ definido na Observação 3 é maximizado. $\mathrm{O}$ restante da demonstração é consequência direta do Teorema 1.

\section{EXEMPLO NUMÉRICO}

Nesta seção é apresentado um exemplo númerico da aplicação das condições de síntese de controladores descentralizados proposta no Teorema 1. Os resultados foram obtidos usando o parser YALMIP (Löfberg, 2004), em conjunto com o solver MOSEK (Mosek, 2017), executado no software MATLAB ${ }^{\circledR}$ R2017a.

O problema considerado trata da estabilização de uma rede elétrica composta apenas por geradores de energia, e é inspirado em (Sadamoto et al., 2016, Example A). O objetivo é verificar a efetividade da abordagem proposta no problema de sincronismo de geradores de energia. A rede de geradores é composta por 7 geradores interconectados de acordo com o grafo apresentado na Figura 1. 


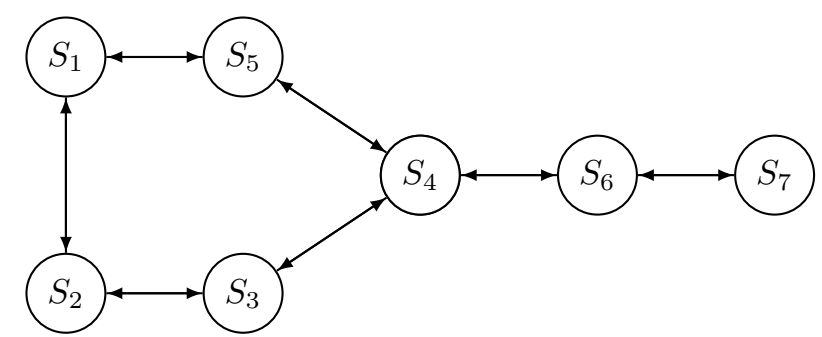

Figura 1. Grafo da rede de geradores de energia.

As equações dinâmicas de cada um dos geradores de energia são descritas da forma:

$$
\left\{\begin{aligned}
\dot{x}_{i 1}(t)= & x_{i 2} \\
\dot{x}_{i 2}(t)= & -\frac{D_{i}}{M_{i}} x_{i 2}(t)-\frac{1}{M_{i}} x_{i 3}(t) \\
& -\frac{1}{M_{i}} \sum_{j \in \mathcal{N}_{i}} Y_{i j} \sin \left(x_{i 1}(t)-x_{j 1}(t)\right), \\
\dot{x}_{i 3}(t)= & -\frac{1}{T_{i}} x_{i 3}(t)+\frac{1}{T_{i}} x_{i 4}(t), \\
\dot{x}_{i 4}(t)= & \frac{1}{K_{i}} x_{i 2}(t)-\frac{R_{i}}{K_{i}} x_{i 4}(t)+\frac{1}{K_{i}} u_{i}(t),
\end{aligned}\right.
$$

em que o vetor de estados $x_{i}(t) \in \mathbb{R}^{4}$ é composto pela diferença em relação a referência do ângulo de fase, pela derivada dessa diferença, pela diferença de entrada de potência mecânica, e pela diferença de posição da válvula de acionamento da turbina; $u_{i}(t) \in \mathbb{R}$ representa o sinal da entrada de controle para o governador do $i$-ésimo gerador; $Y_{i j}$ é a admitância entre o $i$-ésimo e $j$-ésimo gerador e as interconexões não-lineares são definidas como $\varphi_{i j}(x)=Y_{i j} \sin \left(x_{i 1}-x_{j 1}\right) \in \operatorname{co}\left\{0, E_{i(\kappa)} x\right\}, \operatorname{com} \kappa \in \mathcal{I}_{d_{i i}}, \mathrm{e}$ $E_{i i(\kappa)}=\left[\begin{array}{llll}Y_{i j} & 0 & 0 & 0\end{array}\right], \forall i \in \mathcal{I}_{N}$ e $E_{i j(\kappa)}=-E_{i i(\kappa)}$, se $\kappa \in \mathcal{N}_{i}$ e $E_{i j(\kappa)}=\left[\begin{array}{lll}0 & 0 & 0\end{array}\right]$, caso contrário. Os parâmetros $M_{i}$, $D_{i}, T_{i}, K_{i}$ e $R_{i}$ denotam a inércia, o coeficiente de amortecimento, a constante de tempo da turbina, a constante de tempo do governador, e a constante característica de redução na velocidade de referência do governador, respectivamente, com valores iguais para todos os geradores, $T_{i}=0,1, K_{i}=1$ e $R_{i}=0,1, \forall i \in \mathcal{I}_{7}$. Todas as variáveis físicas estão normalizadas.

Todos os geradores possuem limites de atuação, tal que a entrada de controle sofre saturação e $\bar{u}_{i}=5$. A matriz de admitância é simétrica e definida como: $Y_{12}=1,2819$, $Y_{15}=0,7668, Y_{23}=1,0752, Y_{34}=0,8634, Y_{45}=0,2443$, $Y_{46}=0,558$ e $Y_{67}=0,3343$. Os outros parâmetros de cada gerador e condições iniciais são dados na Tabela 1. Além disso, para definir apropriadamente a região de validade do modelo em (19), foi considerado que $x_{i 2}(t) \geq-120 \pi$, o que limita a excursão máxima da diferença da velocidade angular, uma vez que o gerador não pode mudar seu sentido de giro.

Solucionando-se o problema de otimização do Corolário 2, o valor do $\operatorname{det}\left(\mathbf{Q}_{N}\right)^{1 / n_{x}}$ encontrado é 14,3251. Considerando as condições iniciais para $x_{i 10}$ e $x_{i 20}$ dadas na Tabela 1 , e $x_{i 30}=0, x_{i 40}=0, \forall i \in \mathcal{I}_{7}$, as quais pertencem ao domínio de atração, as trajetórias das diferenças dos ângulos de fase e velocidades angulares para cada gerador são ilustradas nas Figuras 2 e 3, respectivamente. A Figura 4 apresenta os sinais das entradas de controle dos geradores de energia.
Tabela 1. Parâmetros dos geradores de energia.

\begin{tabular}{ccccc} 
Subsistema & $M_{i}$ & $D_{i}$ & $x_{i 10}(\mathrm{rad})$ & $x_{i 20}(\mathrm{rad} / \mathrm{s})$ \\
\hline$S_{1}$ & 0.5 & 1.2 & $-0,0702$ & $-0,0401$ \\
$S_{2}$ & 0.3 & 1.0 & $-0,0212$ & 0,1057 \\
$S_{3}$ & 1.0 & 0.3 & 0,1572 & 0,0075 \\
$S_{4}$ & 0.2 & 1.5 & $-0,0718$ & 0,1470 \\
$S_{5}$ & 0.9 & 0.9 & $-0,1841$ & 0,2175 \\
$S_{6}$ & 0.1 & 0.4 & 0,0730 & $-0,1372$ \\
$S_{7}$ & 0.4 & 0.9 & 0,0951 & $-0,0598$ \\
\hline
\end{tabular}

Note que todos os geradores são estabilizados no ponto de operação desejado, mesmo ocorrendo saturação nos seus respectivos sinais de controle. Isso ilustra a eficácia da abordagem de controle proposta, quando empregada em sistemas interconectados por não-linearidades setoriais e sujeitos a saturação.

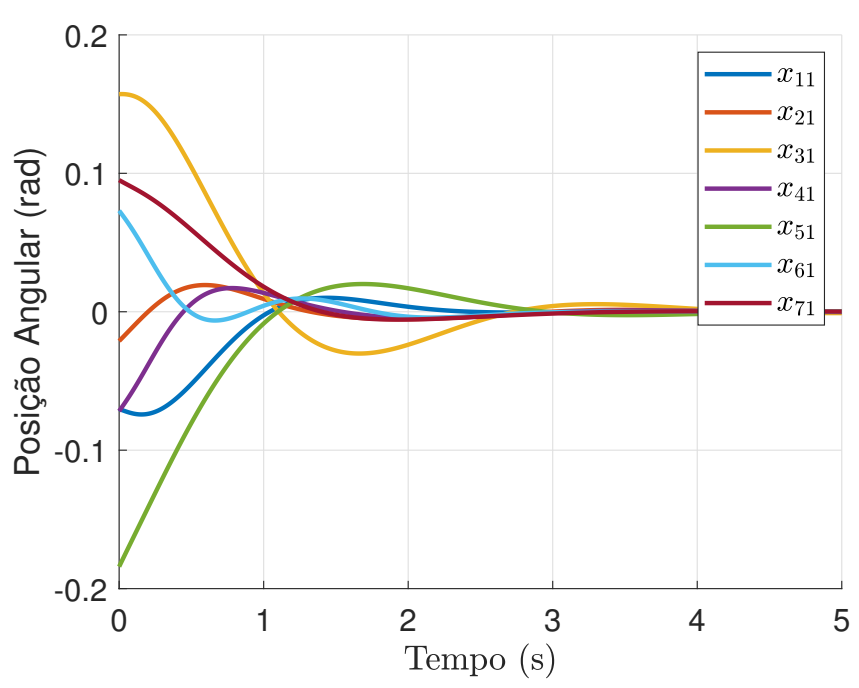

Figura 2. Trajetórias das diferenças dos ângulos de fase dos geradores.

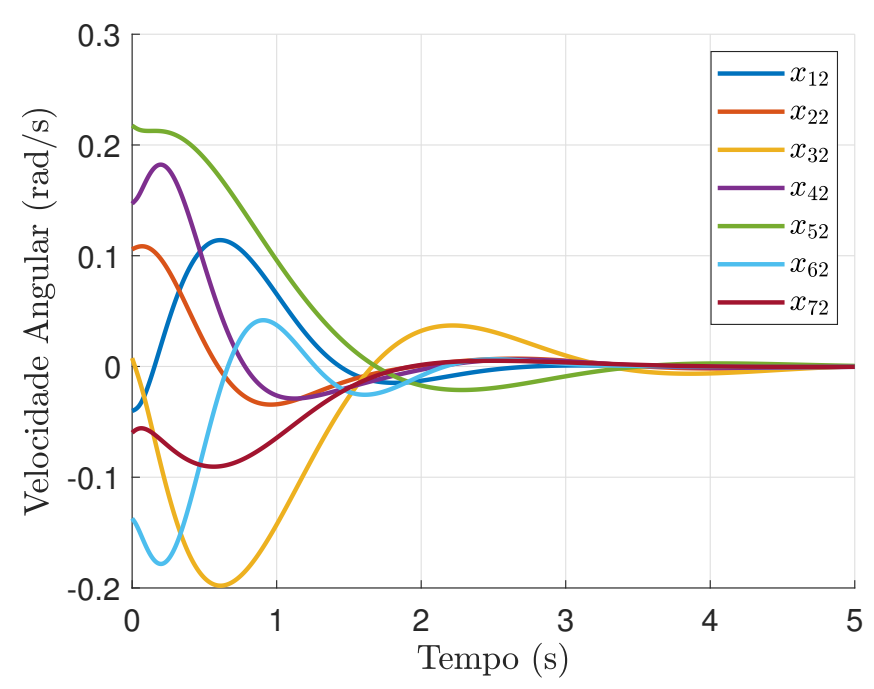

Figura 3. Trajetórias das diferenças das velocidades angulares dos geradores. 


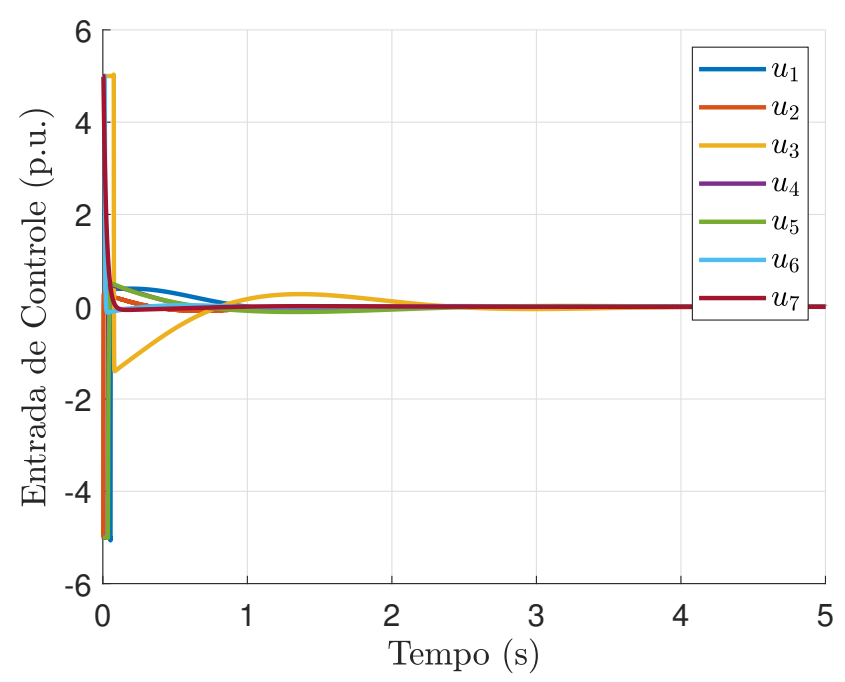

Figura 4. Sinais de controle dos geradores.

\section{CONCLUSÕES}

Neste artigo abordou-se o problema de controle descentralizado para a classe de sistemas a tempo contínuo interconectados por não-linearidades setoriais, sujeitos a restrições nos estados e saturação nos aturadores. Uma função de Lyapunov composta pelo somatório de funções quadráticas foi utilizada para se obter condições suficientes de estabilização, via LMIs. Também foi mostrado que se o problema de otimização é factível, o sistema global em malha fechada é assintoticamente estável e uma estimativa de máxima região de atração elipsoidal pode ser obtida. Finalmente, o problema de sincronismo de geradores de energia interconectados foi usado para ilustrar a eficácia da abordagem proposta para controle descentralizado.

\section{AGRADECIMENTOS}

O presente trabalho foi realizado com apoio da Coordenação de Aperfeiçoamento de Pessoal de Nível Superior - Brasil (CAPES) - Código de Financiamento 001. Os autores agradecem também ao apoio do Conselho Nacional de Desenvolvimento Científico e Tecnológico (CNPq) e da Fundação de Amparo à Pesquisa do Estado de Minas Gerais (FAPEMIG).

\section{REFERÊNCIAS}

Bakule, L. (2008). Decentralized control: An overview. Annual Reviews in Control, 32, 87-98.

Bakule, L. (2014). Decentralized control: Status and outlook. Annual Reviews in Control, 38, 71-80.

Boyd, S., El Ghaoui, L., Feron, E., and Balakrishnan, V. (1994). Linear Matrix Inequalities in System and Control Theory, volume 15. Siam.

Castelan, E., Tarbouriech, S., and Queinnec, I. (2008). Control design for a class of nonlinear continuous-time systems. Automatica, 44, 2034-2039.

Chang, W., Wang, W.J., Li, N.J., Chou, H.G., and Chang, J.W. (2014). $\mathcal{H}_{\infty}$ control synthesis for the large-scale system based on a linear decomposition of nonlinear interconnections. International Journal of Fuzzy Systems, 16, 97-110.
Gonzaga, C., Jungers, M., and Daafouz, J. (2012). Stability analysis of discrete-time Lur'e systems. Automatica, 48, 2277-2283.

Khalil, H.K. (2002). Nonlinear Systems. Prentice Hall, 3rd edition.

Khalili, M., Zhang, X., Polycarpou, M.M., Parisini, T., and Cao, Y. (2018). Distributed adaptive fault-tolerant control of uncertain multi-agent systems. Automatica, $87,142-151$.

Li, Y., Wu, Y., and He, S. (2018). Synchronization of network systems subject to nonlinear dynamics and actuators saturation. Circuits, Systems, and Signal Processing, 1-23.

Löfberg, J. (2004). YALMIP: A toolbox for modeling and optimization in MATLAB. In 2004 IEEE International Symposium on Computer Aided Control Systems Design, 284-289. Taipei, Taiwan.

Mahmoud, M.S. (2010). Decentralized Control and Filtering in Interconnected Dynamical Systems. CRC Press.

Mosek, A. (2017). The MOSEK optimization toolbox for MATLAB manual.

Nguyen, A., Taniguchi, T., Eciolaza, L., Campos, V., Palhares, R., and Sugeno, M. (2019). Fuzzy control systems: Past, present and future. IEEE Computational Intelligence Magazine, 14(1), 56-68. doi:10.1109/MCI. 2018.2881644.

Nguyen, A.T., Laurain, T., Palhares, R.M., Lauber, J., Sentouh, C., and Popieul, J.C. (2016). LMI-based control synthesis of constrained Takagi-Sugeno fuzzy systems subject to $\mathcal{L}_{2}$ or $\mathcal{L}_{\infty}$ disturbances. Neurocomputing, 207, 793-804.

Sadamoto, T., Ishizaki, T., Imura, J., Sandberg, H., and Johansson, K.H. (2016). Retrofitting state feedback control of networked nonlinear systems based on hierarchical expansion. In 2016 IEEE 55th Conference on Decision and Control (CDC), 3432-3437. Las Vegas, USA.

Takagi, T. and Sugeno, M. (1985). Fuzzy identification of systems and its applications to modeling and control. IEEE Transactions on Systems, Man, and Cybernetics, SMC-15, 116-132.

Tarbouriech, S., Garcia, G., Gomes da Silva Jr, J., and Queinnec, I. (2011). Stability and Stabilization of Linear Systems with Saturating Actuators. Springer Science \& Business Media.

Šiljak, D.D. (2007). Large-Scale Dynamic Systems: Stability and Structure. Dover Civil and Mechanical Engineering Series. Dover Publications.

Šiljak, D. and Zečević, A. (2005). Control of largescale systems: Beyond decentralized feedback. Annual Reviews in Control, 29, 169-179. 